\title{
Topology optimized design of a transverse electric higher order mode converter
}

\author{
Frellsen, Louise Floor; Ding, Yunhong; Sigmund, Ole; Frandsen, Lars Hagedorn
}

Published in:

Proceeding of the 2016 IEEE Photonics Conference (IPC)

Link to article, DOI:

10.1109/IPCon.2016.7831071

Publication date:

2016

Document Version

Peer reviewed version

Link back to DTU Orbit

Citation (APA):

Frellsen, L. F., Ding, Y., Sigmund, O., \& Frandsen, L. H. (2016). Topology optimized design of a transverse electric higher order mode converter. In Proceeding of the 2016 IEEE Photonics Conference (IPC) (pp. 793794). IEEE. https://doi.org/10.1109/IPCon.2016.7831071

\section{General rights}

Copyright and moral rights for the publications made accessible in the public portal are retained by the authors and/or other copyright owners and it is a condition of accessing publications that users recognise and abide by the legal requirements associated with these rights.

- Users may download and print one copy of any publication from the public portal for the purpose of private study or research.

- You may not further distribute the material or use it for any profit-making activity or commercial gain

- You may freely distribute the URL identifying the publication in the public portal 


\title{
Topology optimized design of a transverse electric higher order mode converter
}

\author{
Louise F. Frellsen ${ }^{1}$, Yunhong Ding ${ }^{1}$, Ole Sigmund ${ }^{2}$ and Lars H. Frandsen ${ }^{1}$ \\ ${ }^{1}$ DTU Fotonik, Department of Photonics Engineering, Technical University of Denmark, DK-2800 Lyngby, Denmark \\ ${ }^{2}$ DTU Mekanik, Department of Mechanics Engineering, Technical University of Denmark, DK-2800 Lyngby, Denmark
}

The investigation of methods to support the ever increasing demand for data transfer has continued for years; one such method suggested within the field of optical communication, is space division multiplexing (SDM) [1]. Simultaneously the field of photonic integrated circuits (PICs) is being investigated due to attractive features such as high device density and low operating power [2]. For PICs it is necessary with a toolbox of devices and one such of importance to the processing of on chip SDM is the mode converter. Several schemes have been used to realize such devices [3] [4]. In this paper we present the possibility of employing topology optimization (TO) to design a device that allows for reversible conversion between the transverse electric fundamental even $\left(\mathrm{TE}_{0}\right)$ mode and the second higher order odd mode $\left(\mathrm{TE}_{2}\right)$. Topology optimization is an iterative inverse design process, where repeated finite-difference time-domain (FDTD) calculations are made in accordance with a sensitivity analysis. This is done using a software package which has been developed in house, and which has shown to deliver low loss designs with a controllable bandwidth in addition to small device footprints [5]. The design is made for fabrication in silicon-on-insulator (SOI) and previous work has shown excellent concordance between simulations and experimental results when employing 3D TO [6].

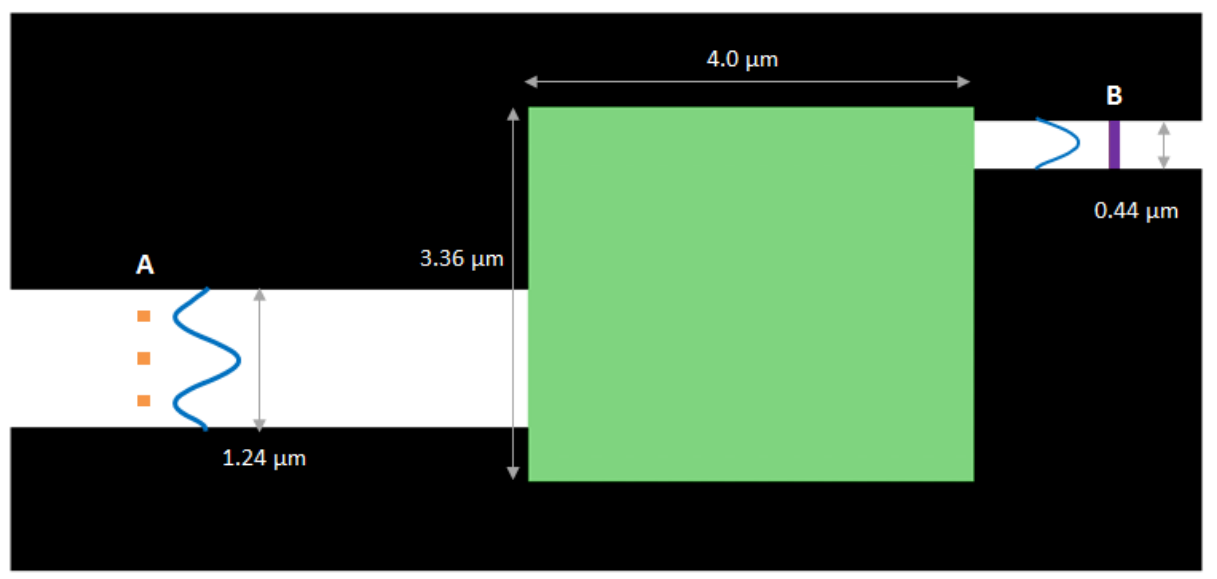

Figure 1 Starting point structure for topology optimization of the mode converter made in silicon (white) and air (black) on a buffer region of silica (not shown). A second higher order odd transverse-electric mode is excited (orange) at the position $A$, and is to be converted to the fundamental transverse-electric mode at the objective (purple) at position B. This is done by altering the material distribution in the design domain (green).

In Figure 1 the starting point structure for the TO is shown. The objective of the optimization is converting the $\mathrm{TE}_{2}$ mode, excited at position $\mathrm{A}$ (orange) in the wide waveguide, to the $\mathrm{TE}_{0}$ mode output in the narrow waveguide. The TO, redistributing the material, is performed in the $4 \mu \mathrm{m} \times 3.6 \mu \mathrm{m}$ design domain (green) to attain the objective intensity distribution at position B (purple). The design is obtained after approximately 200 iterations.

The optimization is performed in $3 \mathrm{D}$, as this has been found to be necessary for correspondence with fabricated structures. The resolution was $40 \mathrm{~nm}$ per pixel and perfectly matched absorbing layers were used as boundary conditions surrounding the structure. The $\mathrm{TE}_{2}$ mode was excited by three separate sources with spectral widths of $280 \mathrm{~nm}$ (full-width half-maximum) centered at $1580 \mathrm{~nm}$ placed at the extrema of the mode profile. The silicon slab has a thickness of $340 \mathrm{~nm}$ with a relative permittivity of $\varepsilon_{\mathrm{Si}}=3.418$ and the silica buffer underneath has a relative permittivity of $\varepsilon_{\mathrm{SiO} 2}=1.444$, from above the structure is surrounded by air. The design domain is restricted to remain uniform in the vertical direction to render fabrication possible.

In Figure 2 the final design is presented along with the structure overlaid with a simulation of the out of plane $\mathrm{H}$-field $\left(\mathrm{H}_{\mathrm{z}}\right)$. The functionality of the design is clear from Figure 2 and is quantified by the 
recorded flux presented in Figure 3(a), the $\mathrm{TE}_{0} / \mathrm{TE}_{2}$ extinction ratio is $>19 \mathrm{~dB}$. Figure 3(b) shows the 3D FDTD calculated transmission spectrum for the $280 \mathrm{~nm}$ bandwidth of the source revealing a loss of $<0.5 \mathrm{~dB}$ near the center wavelength and a $3 \mathrm{~dB}$ bandwidth of $\sim 113 \mathrm{~nm}$, the data has been normalized to a spectrum recorded for a straight waveguide.
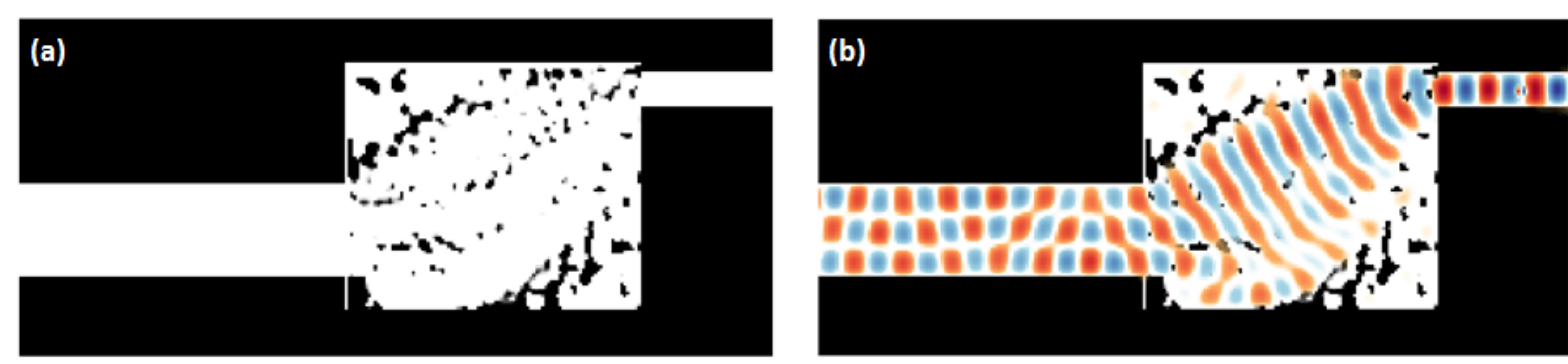

Figure 2 The design obtained through 3D topology optimization of the structure shown in Figure 1 depicted in black (air) and white (silicon) (a) without and (b) with the 3D FDTD-calculated propagation of the $H_{z}$-field through the mode converter at $1580 \mathrm{~nm}$.

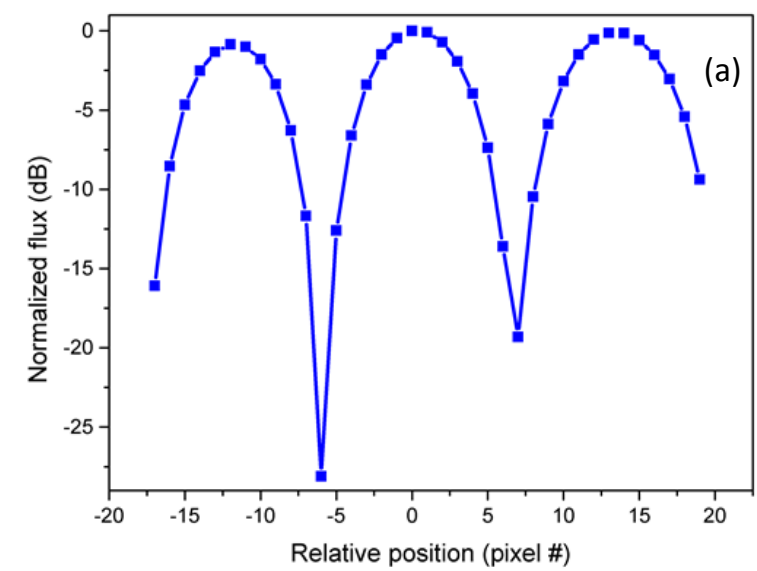

(a)

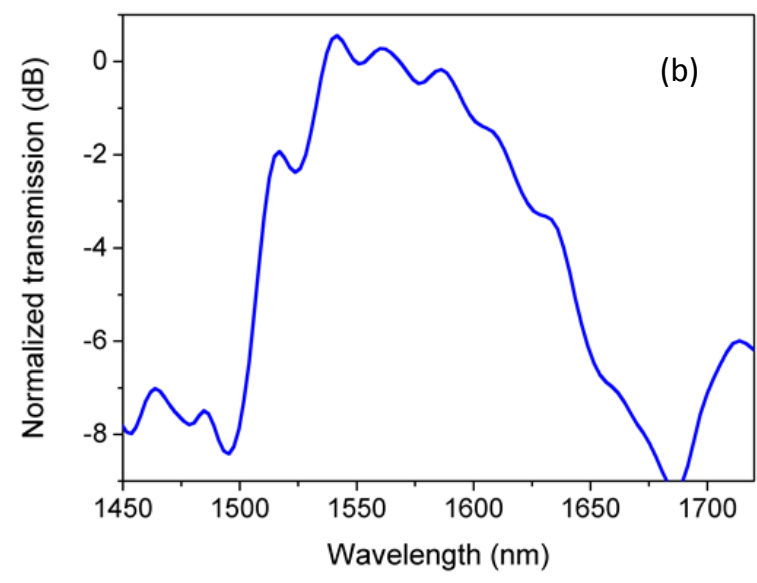

(b)

Figure 3 (a) Power flux recorded at position A in Figure 1 when exciting a fundamental mode at position B, shown as a function of relative position across the waveguide with $0 \mathrm{~nm}$ corresponding to the center of the waveguide. (b) $3 D$ FDTDcalculated transmission spectrum of the $T E_{0}$ mode obtained after conversion. The spectrum is normalized to that of a $T E_{0}$ mode in a straight waveguide.

Previous work has demonstrated that 3D topology optimized designs are readily fabricated using ebeam revealing experimental results in correspondence with the simulated data [7]. Furthermore this design confirms that the method can, as previously claimed, be extended to function for higher order modes without significant increase in footprint or transmission losses.

[1] M. Lipson, "Guiding, Modulating, and Emitting Light on Silicon - Challenges and Opportunities," Journal of Lightwave Technology, vol. 23, no. 12, pp. 4222-4238, 2005.

[2] W. S. Zaoui, A. Kunze, W. Vogel, M. Berroth, J. Butschke, F. Letzkus and J. Burghartz, "Bridging the gap between optical fibers and silicon photonic integrated circuits," Optics Express, vol. 22, no. 2, pp. 1277-1286, 2014.

[3] M. Liu, D. A. B. Miller and S. Fan, "Ultra-compact photonic crystal waveguide spatial mode converter and its connection to the optical diode effect," Opt. Express, vol. 20, no. 27, pp. 28388-28397, 2012.

[4] Y. Ding, J. Xu, F. Da Ros, B. Huang, H. Ou and C. Peucheret, "On-chip two-mode division multiplecing using tapered directional coupler.based mode multiplexer and demultiplexer," Optics Express, vol. 21, no. 8, 2013.

[5] Y. Elesin, B. S. Lazarov, J. S. Jensen and O. Sigmund, "Time domain topology optimization of 3D nanophotonic decives," Photon. Nanostruct. Fund. Appl., vol. 12, no. 1, pp. 23-33, 2014.

[6] L. H. Frandsen, Y. Elesin, L. F. Frellsen, M. Mitrovic, Y. Ding, O. Sigmund and K. Yvind, "Topology optimized mode conversion in a photonic crystal waveguide fabricated in silicon-on-insulator material," Optics Express, vol. 22, no. 7, 2014.

[7] L. F. Frellsen, L. H. Frandsen, Y. Ding, Y. Elesin, O. Sigmund and K. Yvind, "Topology optimized design for silicon- 